\title{
Design and Implementation of Logical Topology in Sensor Network for an Industrial Stack Monitoring
}

\author{
P.Velmani \\ Asst.Prof in Computer Science \\ The M.D.T. Hindu College \\ Tirunelveli, Tamilnadu, India
}

\author{
K.Ramar \\ Prof \& Head \\ Department of CSE \\ Srividya College of Engineering and Technology, \\ Virudhunagar, Tamilnadu, India
}

\begin{abstract}
Wireless Sensor Network (WSN) has gained worldwide attention in recent years. The design of a WSN depends significantly on the application. In sensor network design, physical and logical topology plays a major role. Transfer information in real time means, to provide a required result in a bounded time. Large-scale sensor networks will not be able to simply send all the raw sensed sample data to a sink. This paper presents an approach of simulation of various sampling techniques (logical topology) of industrial stacks emission and also defines a network with rough node deployment. The simulation of the sampling techniques is carried out using Castalia simulator based on OMNET++ language.
\end{abstract}

\section{General Terms}

Computer Science - Wireless Sensor Network

\section{Keywords}

Wireless Sensor Network, Stack monitoring, sampling technique, Castalia Simulator.

\section{INTRODUCTION}

In recent years, Wireless Sensor Network (WSN) has evolved worldwide attention due to the importance of monitoring in harmful / hazardous environment. The development of WSN has its roots in military research and applications in monitoring the battle field. Today, they are widely used in environmental monitoring and protection of nature and man. More applications are monitoring widespread fire, control the level of water and air pollution in an industry, atmospheric collection of information (humidity, pressure) and monitoring wild life protected areas.

The design of a WSN depends significantly on the application. To construct an efficient air pollution monitoring sensor network in an industrial area, application domain space and network domain space are the two avenues to be considered [1, 2]. Without through knowledge of the application domain, one cannot design an effective sensor network. In sensor network design, physical and logical topology plays major role. The physical topology refers to the configuration of connection between peripherals involved in the network like sensor, computer and transmission media [3]. The logical topology is a method used to pass information between them.

In an industrial point source, emissions emanate from a very small opening such as a stack or vent. Stacks usually emit hot gases forcefully into the atmosphere at a fixed height above ground level. Samples should be collected under all environmental conditions (temperature, wind speed etc.) that affect pollution levels. The main aim of the work is to test some sampling technique in simulation before moving in to real systems. The purpose of monitoring is collecting data systematically and used for further analysis. So, this paper presents an approach of simulation of monitoring continuously using various sampling technique (logical topology) - periodic time sampling, level sampling, time interval sampling in industrial stack emission based on source release parameters like stack height and stack diameter and also defines a network with rough node deployment. The simulation of the sampling technique is carried out with the simulator Castalia based on OMNET++ (Object-oriented Modular NETwork) language [4].

\section{NODE DEPLOYMENT}

One of the design optimization strategies in WSN is to find out the place of sensor nodes in order to meet the desired performance goals. The placement strategies are static or dynamic and structured or unstructured. The static or dynamic strategies depend on whether the optimization is performed at the time of deployment or while the network is operational. In a structured WSN, sensor nodes are deployed in a pre-planned manner whereas in an unstructured WSN, sensor nodes are deployed in an ad hoc manner $[5,6]$.

In a structured with static sensor network, sensor nodes are pre-determined to be placed at fixed locations. The choices are grid placement, optimal placement, 2Dimension (D) and 3D placement models. In some real world application scenario, the deployed sensor network operates over a 3D volume rather than in a 2D area. Deployment of WSNs for surveillance of terrains, study of underwater ecosystem, space monitoring and explosion, monitoring air pollution at mountain etc are examples of such application. Industrial Stack monitoring WSN is also one such scenario. This paper defines the 3D node location for stack monitoring. 3D Localization scheme, which is identification of sensor nodes position, is also reported in few papers $[7,8]$.

One of the topological issues of WSN is coverage topology. Coverage problem reflects how well an area is monitored or tracked by sensors. The coverage and connectivity problems in sensor network have received considerable attention in the research community [9]. In industrial Stack monitoring WSN, the nodes can be placed as per the norms of central pollution control board, India [10].

The sampling site or plane is from a disturbance to emission gas flow, such as a bend or a fan. In sampling plane, sensor must be fixed at a point known as sampling point. As per norms, sampling point must be located at least $8^{*}$ stack diameter height from downstream of a bend and $2 *$ stack diameter height upstream from stack exit, as shown in Figure 1. A number of locations may be used as a sampling plane. But smaller number of sampling diameters or points may be adequate [11]. 


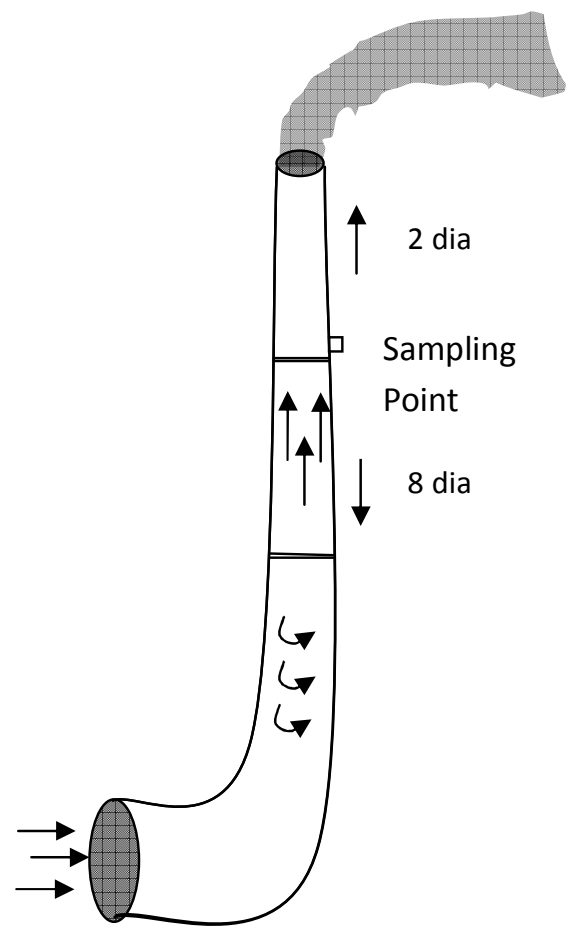

Figure 1: Sampling point in a stack.

The implementation of a WSN is prone to multiple factors and is not guaranteed as easily as the cable. The provision of environmental space, the distance between nodes, electromagnetic interference and too many other factors determine the quality of the connection and achieving a wireless communication network [12]. Before installing a network, it is necessary to ensure a degree that it will be successful and functional. For this purpose, this paper defines a network with rough node deployment and checks the various sampling mechanism.

\section{SIMULATION SCHEMES}

Air monitoring sampling technique available is continuous sampling and short or spot sampling [13]. Spot sampling cannot give adequate data on the nature and the magnitude of an air pollution problem [14]. Continuous sampling or transfer information in real time means, to provide a required result in a bounded time. Effective sampling rate is one of the system evaluation metrics. Large-scale sensor networks will not be able to simply send all the raw sensed data to a sink. Energy and channel capacity constraints will require as much localized processing as possible, delivering only a (small) high-level sensing result. A perfect system will reduce as much data as possible as early as possible. With this aim, various schemes of continuous sampling data transmission technique are evaluated using Castalia-2.3b, a state of the art WSN simulator based on the OMNET++ platform.

The simulation steps carried out are

(1) Sampling rate - the study of reporting sensed data from single source to a sink

(2) Scalability - studying the sampling behavior in a small network (four sources and a sink) in an industry
The schemes of data transmission technique are defined in the valueReporting handleMessage () method. Castalia has a modular structure. Each one of the modules contains one or more parameters that affect its behavior. These parameters have types and so the user can only assign specific types of values to them either directly in the NED files or in the configuration file omnetpp.ini or in the ini files in the Castalia/Simulations /Parameter_Include_Files directory [15, 16]. The following files are included for the study.

include../Parameter_Include_Files/physical

Process_0_node0_asssignedValue40.ini

include./Parameter Include Files/resource

Mgr_2AAbatteries.ini

include../Parameter_Include Files/nodeSensor

DevMgr_Pollution.ini

include../Parameter Include Files/WChannel/

Additive_Interference_Model/WChannel_

Realistic.ini

include../Parameter_Include_Files/Radio/

TelosB_CC2420/radio_CC2420.ini

include./Parameter_Include_Files/MAC_

just_carrierSense.ini

include./Parameter_Include_Files/Routing simpleTree.ini

\subsection{Sampling Rate}

Stack monitoring through single source is carried out based on two schemes. For one source, sink may be fixed at $10 \mathrm{~km}$ radius in TelosB model. For example if source is at $(25,25,80)$ then sink in floor may be fixed from $(15,15,2)$ to $(35,35,2)$.

\subsubsection{Scheme - A}

Sampling period or sampling interval is the time between samples. The evaluation of peak and average pollution concentrations over definite time interval is sometimes needed. For that purpose sampling may be taken place over definite time interval, for example APP_SAMPLE_INTERVAL 1000(s). The part of the handle message () method and output is shown in Figure 2.

\subsubsection{Scheme- $B$}

The part of the handle message () method in Figure 3 is to receive the values that is greater than the defined pollution threshold level. This case is to test the reaction when a node is an alert case.

\subsection{Scalability}

The sampling pattern of small network is studied by constructing a network with four sources and a sink. The node locations of the four sources are $(13,13,40),(25,25$, $80),(50,50,120),(75,75,200)$ and sink may be fixed at from $(25,25,2)$ to $(35,35,2)$. Since this location is suitable to receive values from all four sources in TelosB model. 


\begin{tabular}{|c|}
\hline $\begin{array}{l}\text { \#define APP_SAMPLE_INTERVAL } 1000 \\
\text { case APP_SELF_REQUEST_SAMPLE: } \\
\text { \{requestSampleFromSensorManager0; } \\
\text { scheduleAt(simTime()+DRIFTED_TIME } \\
\text { (APP_SAMPLE_INTERVAL), } \\
\text { new App_ControlMessage("Application self } \\
\text { message(request sample)", } \\
\text { APP_SELF_REQUEST_SAMPLE)); } \\
\text { break;\} }\end{array}$ \\
\hline $\begin{array}{l}T=6.78788, \text { Sink(Node }[0]) \\
\text { received from: } 1 \quad \text { value }=39.999\end{array}$ \\
\hline $\begin{array}{l}\mathrm{T}=1006.81, \text { Sink(Node }[0]) \\
\text { received from: } 1 \text { value }=39.911\end{array}$ \\
\hline $\begin{array}{l}\mathrm{T}=2006.82, \text { Sink }(\text { Node }[0]) \\
\text { received from: } 1 \quad \text { value }=39.805\end{array}$ \\
\hline $\begin{array}{l}T=85008, \text { Sink(Node[0]) } \\
\text { received from: } 1 \quad \text { value }=39.877\end{array}$ \\
\hline $\begin{array}{l}\mathrm{T}=86008, \operatorname{Sink}(\text { Node }[0]) \\
\text { received from: } 1 \quad \text { value }=\mathbf{4 0 . 1 1 5}\end{array}$ \\
\hline
\end{tabular}

Figure 2: Sampling at the rate of 1000s.

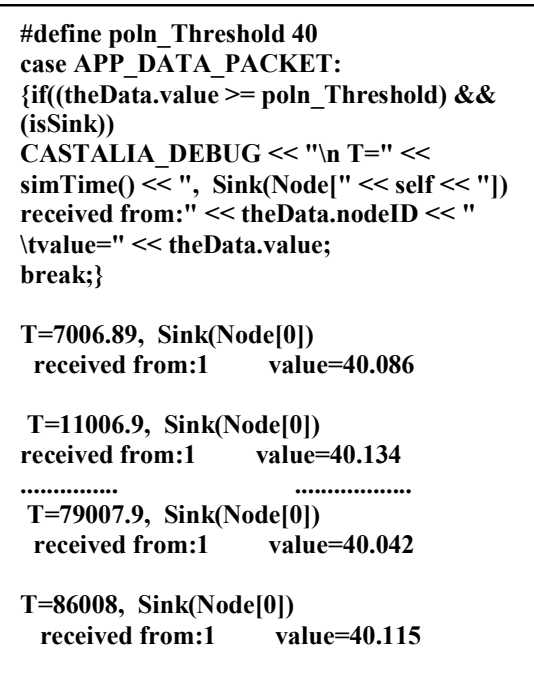

Figure 3: Sampling greater than Threshold value

\subsubsection{Scheme- $A$}

The evaluation of peak and average concentrations over definite time interval in an industry may be sometimes needed. Samples representing a full-day (86400s) exposure shall be collected for evaluating or assessing the Time Weighted Average (TWA) concentration. For that purpose, sampling over definite time interval for all nodes in a network may be carried out in APP_SAMPLE_INTERVAL 1000(sec). The part of the handle message () method is shown in Figure 4.

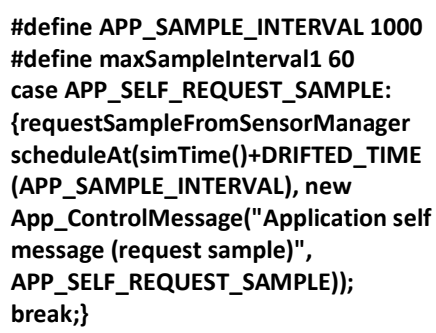

Figure 4: Periodic time sampling.

The sensed values are assigned through physical process_0 node 0 assingnedvalue 40 .ini file. The values are $40,100, \overline{1} 5 \overline{0}$ and 80 for the nodes1, 2, 3 and 4 respectively. Node 0 is considered as a sink. The graphical representation of the output for all four nodes is shown in Figure 5.

\subsubsection{Scheme- $B$}

Most industrial operations have variable process conditions which change the characteristics and quantities of pollutants discharged. To provide a detailed record of large emission source, the sampling interval may be configured frequently; otherwise the larger interval sampling rate is sufficient. To study the multiple sample rates, the third node is configured to send values at 1000 s interval and other sources are configured to send values at $2000 \mathrm{~s}$ interval. The part of the handle message () method is shown in Figure 6.

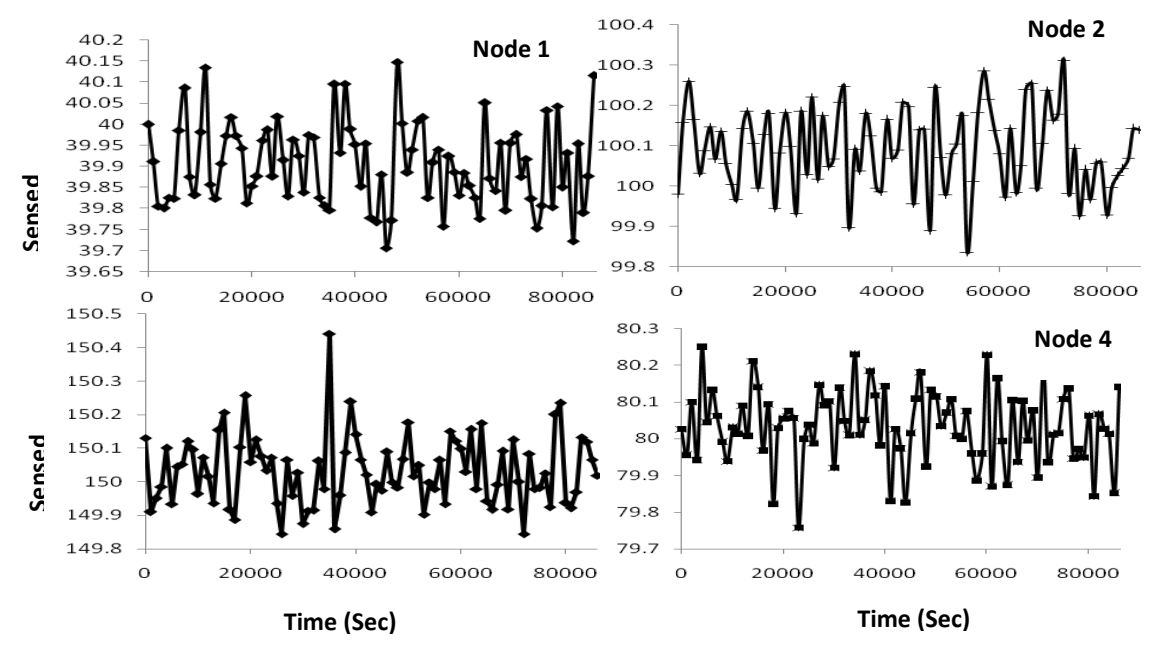

Figure 5: Sampling at the rate of $1000 \mathrm{~s}$. 
\#define APP_SAMPLE_INTERVAL1 1000

\#define APP_SAMPLE_INTERVAL2 2000

\#define maxSampleInterval1 60

case APP_SELF_REQUEST_SAMPLE:

\{requestSampleFromSensorManager();

if(self $==1)$

scheduleAt(simTime()+DRIFTED_TIME(APP S

AMPLE_INTERVAL1), new

App_ControlMessage("Application self message (request sample)",

APP_SELF_REQUEST_SAMPLE));

else

scheduleAt(simTime()+DRIFTED_TIME(APP_S AMPLE_INTERVAL2), new

App_ControlMessage("Application self message

(request sample)",

APP_SELF_REQUEST_SAMPLE));

break;

Figure 6: Multiple sample rates.

\subsubsection{Scheme- $C$}

The part of the handle message () method in Figure 7 is to receive the values that is greater than the defined pollution threshold. The output shows only node 3 is sensing the threshold values.

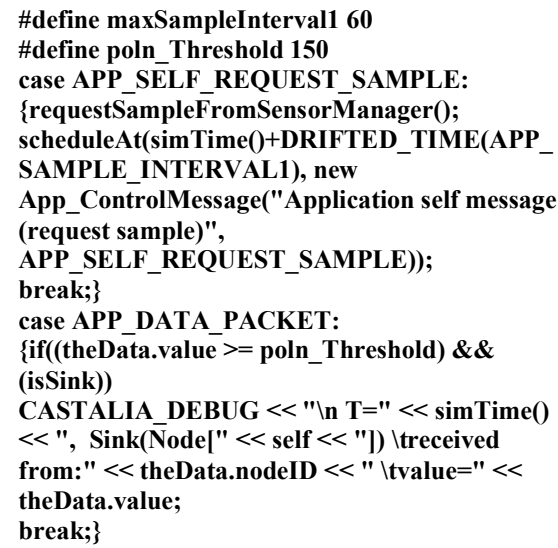

Figure 7: Value Sampling.

The graphical representation of the output of node 3 above threshold value is shown in Figure 8-a. For the sake of comparison the values received both above and below threshold is shown in Figure 8-b. The solid line represents threshold line.

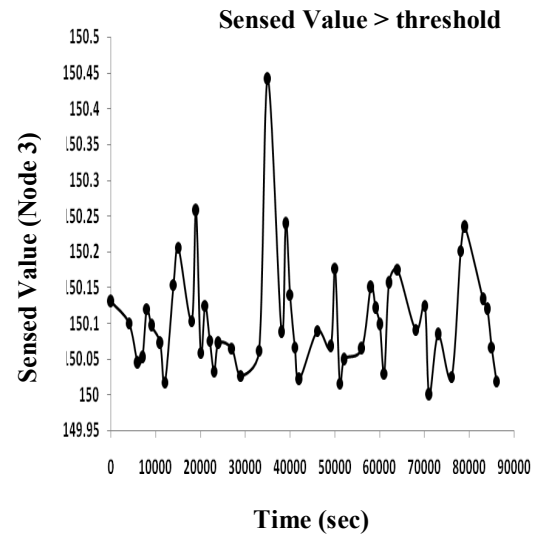

Figure 8-a: Node 3 sensed value greater than threshold.

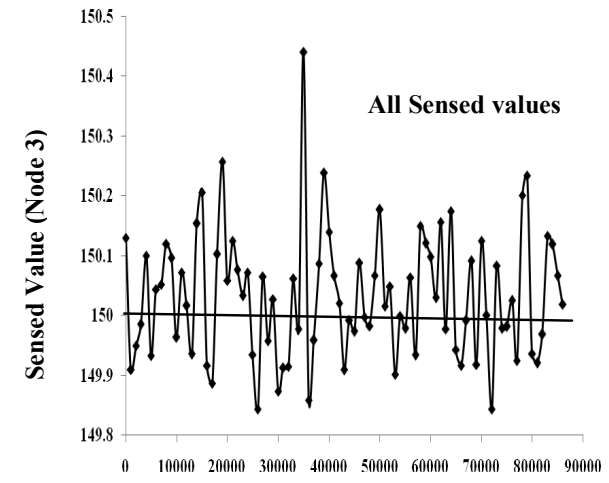

Time (sec)

Figure 8-b: Node 3 sensed interval 1000s.

\subsubsection{Scheme- $D$}

To measure pollution in various time periods like morning, noon, evening, start and close time of the particular shift, shut down time if any may also be carried out to study the variation in pollution level. For example, consider the first shift starts at night 12 o'clock that is at 28800 seconds. Figure 9 shows the part of the handle message () method and output for sampling required from the time period 15 minutes before - 27900 seconds to 15 minutes after - 29700 seconds.

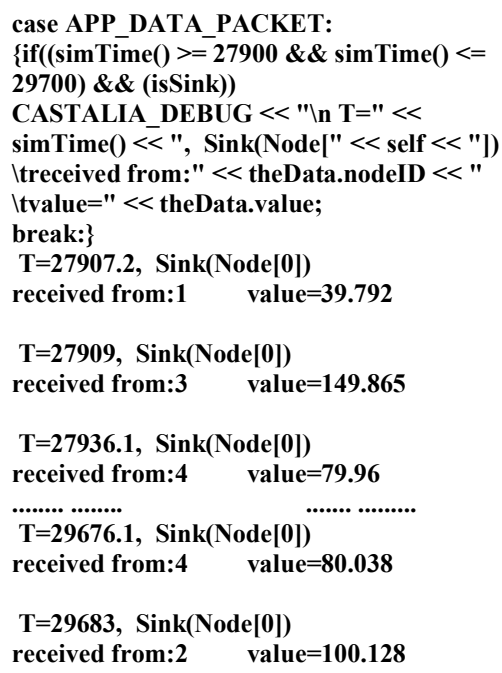

Figure 9: Time interval sampling.

\section{RESULTS OF SIMULATION SCHEMES}

- To achieve certain requirements, resource management particularly energy resources and sensor's RAM Memory is important for WSNs. The value of spent energy obtained for each node in the network, in a simulation time fixed to 86400 seconds ( 1 day) is given below. Energy consumption is based on the time the radio is on (either listening, transmitting or receiving). If two nodes have the same on time then they will have the same energy consumed. 
Node [0] spent energy: $\mathbf{5 8 7 5 . 2 0}$

Node [1] spent energy: $\mathbf{5 8 7 5 . 1 9}$

Node [2] spent energy: $\mathbf{5 8 7 5 . 1 9}$

Node [3] spent energy: $\mathbf{5 8 7 5 . 1 9}$

Node [4] spent energy: $\mathbf{5 8 7 5 . 1 9}$

- The main aim of different sampling technique is to collect only valuable data and hence the amount of data transmitted to the sink, RAM Memory used and transmission channel utilization is reduced. For example, in scheme 3.2.2, number of times sample values received from node 3 is 87 , and from other nodes half of the times that is only 44 times. It is assumed that node 3 is a most polluted unit of an industry under keen inspection.

- $\quad$ Figure 8-b shows one day simulation of sensed value of node 3 at an interval 1000s. Number of times sensed value received is 87 . But all these sensed values are not important if it is tolerable pollution range. In that case only values greater than particular threshold level may be passed to the sink. The values above the threshold line, as shown in figure 8-b is received as shown in figure 8-a. By this scheme instead of 87 times, the number of times sensed values received is minimized to 49.

- Based on industrial scenario, the various sampling techniques like periodic time sampling, level sampling and time interval sampling can be used to determine the concentration of air pollution through stack and these may be taken as guidance to compare results in different ways.

\section{CONCLUSION}

Effective sampling rate is one of the system evaluation metrics. This paper deals with optimization in sampling techniques namely periodic time sampling, level sampling, time interval sampling of stack monitoring based on certain source release parameters like stack height, stack diameter. From this simulation study of sampling methods, it is concluded that the amount of data transmitted to the sink, RAM memory used and transmission channel utilization are reduced. Furthermore, the optimization in sampling may be possible in terms of the parameters namely node number (self), sensed value (theDatavalue) and simulation time (simTime). These results will enable investigators to conduct various types of air pollution sampling, quantify contamination levels, and conduct risk assessments.

\section{REFERENCES}

[1] Kay Romer, Friedemann Mattern and Eth Zurich. Dec 2004. The design space of wireless sensor networks. IEEE Wireless communications, p. 54.

[2] Velmani, P., and Ramar, K. 2009 Application domain space of sensor network in industrial air pollution monitoring - A study, proceeding of ICONCT pp. 197202
[3] Ian F.Akyildiz, Weilian Su, Yogesh Sankarasubramaniam, and Erdal Cayirci, Georiga Institute of Technology, Aug 2002. A survey on sensor networks. IEEE communications Magazine, pp. 102-114.

[4] Velmani, P. and Ramar, K. Feb 2011. Castalia: Suitable Simulator for WSN Applications, RAC journal of research, Vol 1, No 2, ISSN:2230 - 7362., pp.59-62.

[5] Jennifer Yick, Biswanath Mukherjee, and dipak Ghosal. 2008 Wireless sensor network survey. Elsevir,Computer networks 52 (2008), pp:2292-2330.

[6] Mohamed Younis., and Kernal Akkaya, 2008, Strategies and techniques for node placement in wireless sensor networks: A survey. Elsevier, Science direct, Ad Hoc Networks 6, pp:621-655.

[7] Shi, Q., Huo, H., Fang,T., and Li, D. 2009. A 3D node localization scheme for wireless sensor networks, IEICE Electronics express, Vol 6, No 3, pp: 167-172.

[8] Vibha Yadav, Manas Kumar Mishra, Singh, A.K., and Gore, M.M. Dec 2009. Localization Scheme for Three Dimensional wireless Sensor Networks Using GPS Enabled Mobile sensor nodes, International Journal of Next - Generation Networks (IJNGN), Vol 1, No 1, pp:60-72.

[9] Muthukarapagam,M., Neveditta,Y., and Neduncheliyan, S. 2010. Design issues, Topology issues, Quality of service support for wireless sensor networks: Survey and research challenges, International Journal of Computer Applications (0975 - 8887), Vol1, No6.

[10] Stack monitoring - material and methodology for isokinetic sampling, Central pollution control board, Ministry of environment and forest notification, chapter 1,2009 .

[11] Haizhuang Kang, Qingping Yang, Member, IEEE, Clive Butler, Tuqiang Xie, and Fabrizio Benati, Apr 2000 .Optimization of Sensor Locations for Measurement of Flue Gas Flow in Industrial Ducts and Stacks Using Neural Networks IEEE Transactions on Instrumentation and Measurment, Vol. 49, No. 2, pp 228-232.

[12] Alessandra Flammini, Paolo Ferrari, Daniele marioli, Emiliano Sisinni, Andrea Taroni. 2009. wired and wireless sensor networks for industrial applications. Elsevir, Microelectronics journal Vol 40, pp 1322-1336.

[13] TamilNadu Pollution Control Board Plan outlay www.tnpcb.gov.in.

[14] Aaradhana Salpekar and Kadambari Sharma, Bharatia Khadi Gramodyog Vikas Abhikaran, New Delhi 2006 "Encyclopedia of Ecology and Environment,", Vol.8, pp.36- 37 .

[15] Castalia, A simulator for Wireless Sensor Networks and Body area Networks, volume 2.3, User Manual, 2009.

OMNeT++, Discrete Event Simulator system, Version 3.0, User Manual, 2004. 